Check for updates

Cite this: RSC Adv., 2017, 7, 27678

\title{
Facile single-step bioconjugation of the antifungal agent caspofungin onto material surfaces via an epoxide plasma polymer interlayer $\dagger$
}

\author{
Thomas D. Michl, id $\ddagger^{\mathrm{a}}$ Carla Giles, $\stackrel{\ddagger}{\mathrm{a}}^{\mathrm{a}}$ Alasdair T. Cross, ${ }^{\mathrm{b}}$ Hans J. Griesser ${ }^{\mathrm{a}}$ \\ and Bryan R. Coad (D) *ac
}

\begin{abstract}
We report a facile, one-step, aqueous surface bioconjugation approach for producing an antifungal surface coating that prevents the formation of fungal biofilms. By direct reaction between surface epoxide groups and amine groups on caspofungin, it avoids the use of secondary chemicals. The coating withstands washing with detergent and reduces the growth of the fungal pathogens Candida albicans by log 6 and Candida glabrata by log 3. Importantly, we show that surface adsorption of albumin does not inhibit the activity of this antifungal coating.
\end{abstract}

Received 6th April 2017

Accepted 14th May 2017

DOI: 10.1039/c7ra03897f

rsc.li/rsc-advances

to the biomedical device at the site of implantation, and avoid systemic drug administration. However, it is important to select a drug that retains activity even after surface attachment, which would suggest avoiding drugs that target endoplasmic processes. It is also essential to verify that all the drug molecules are indeed covalently bound and not just physisorbed or indiffused, to avoid possible misleading interpretation of observations.

A potential pitfall, however, might be that an immobilised surface layer is likely to rapidly become buried underneath adsorbing proteins when exposed to body fluids, and hence approaching microbial cells would settle onto a protein layer and no longer experience the drug molecules underneath. Perhaps this might be a reason for the lack of success in vivo of candidate coatings; in vitro tests are usually performed in microbial broth and thus do not replicate the clinical situation. ${ }^{5}$ Accordingly, we have in this study also investigated the antifungal performance of coatings after they had been exposed to an albumin solution.

Caspofungin seems an ideal candidate for a covalently immobilised antifungal coating due to its inhibition of the 1,3$\beta$-(D) glucan synthase proteins which are involved in cell wall synthesis. ${ }^{6}$ While covalent surface attachment of caspofungin has been reported previously, ${ }^{7}$ approaches such as carbodiimide chemistry or reductive amination ${ }^{8}$ entail the use of catalysts or secondary chemicals, which complicate waste treatment in the scale-up of manufacturing processes. A direct reaction between the drug molecule and complementary reactive surface groups would be much preferable; this is achievable via the spontaneous reaction between amine and epoxide groups. We have previously developed a plasma polymerisation approach with an epoxide process vapour to creating thin polymeric films with surface epoxide groups. ${ }^{9}$ The ring opening of surface
${ }^{a}$ Future Industries Institute, University of South Australia, Mawson Lakes, SA 5095, Australia.E-mail: bryan.coad@adelaide.edu.au

${ }^{b}$ The University of Bath, Claverton Down, Bath BA2 7AY, UK

${ }^{c}$ School of Agriculture, Food and Wine, University of Adelaide, Adelaide, SA 5005, Australia

$\dagger$ Electronic supplementary information (ESI) available. See DOI: 10.1039/c7ra03897f

\$ These authors contributed equally. 
epoxide groups by amines under neutral $\mathrm{pH}$ conditions would allow facile one-step immobilisation of caspofungin (and other molecules with amine groups) under gentle aqueous conditions via an $\mathrm{S}_{\mathrm{N}} 2$ reaction (Scheme 1). There are no by-products that would need to be considered in process scale-up.

The deposition of an epoxide-functionalised plasma polymer interlayer for covalent bonding of bioactive molecules also offers the advantage that this layer can be applied onto a wide range of materials, making the present antifungal coating approach transferable to a wide range of materials and biomedical devices. ${ }^{10}$ Plasma polymerisation of allyl glycidyl ether (AGE) by using a pulsed plasma approach has been shown to produce coatings with good structural retention of reactive epoxide groups. ${ }^{9,11}$ Optimal conditions consisted of depositing an initial layer for 1 minute under continuous wave (CW) conditions to improve the adhesion to the substrate, followed immediately by pulsing the plasma for 2 minutes to maximize surface epoxide groups ( $\mathrm{CW}+$ pulsed). ${ }^{9}$

Fig. 1 depicts the thickness of the CW layer being $59.9 \pm$ $1.1 \mathrm{~nm}$ after $1 \mathrm{~min}$ of deposition. Pulsing for an additional $2 \mathrm{~min}$ increased the overall thickness to $84.4 \pm 1.2 \mathrm{~nm}$. This slow growth under pulsed conditions was expected and observed in other publications due to the decreased timeaveraged power input, ${ }^{12}$ which in turns leads to improved epoxide group retention.

We explored various washing procedures and how they affected the stability of the AGE plasma polymer (AGEpp) coating. Water, ethanol, acetone and SDS were used to wash samples of the CW AGEpp as well as CW + pulsed AGEpp for 15 minutes. The thickness of the coatings was measured before and after washing to quantify the washing procedure's effects on coating thickness, such as swelling or dissolution.

As shown in Fig. 2, washing with water alone had a negligible effect on the thickness of the plasma polymer layer. The CWonly coating swelled by less than $1 \mathrm{~nm}$ and the $\mathrm{CW}+$ pulsed coating lost a marginal $3 \mathrm{~nm}$ of thickness, presumably due to the dissolution of soluble lower-molecular-weight material from the film. The CW coating in ethanol experienced a $13 \%$ decrease in thickness and the CW + pulsed coating $16 \%$. Similarly, washing with acetone led to a decline in thickness of $16 \%$ and $17 \%$, respectively. The thickness decrease after washing by both
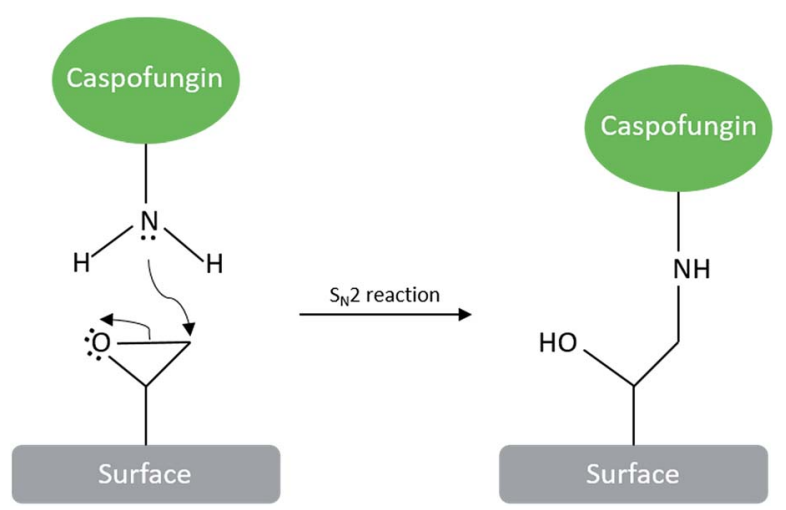

Scheme 1 Formation of interfacial amine bond by reacting caspofungin with epoxide surfaces.

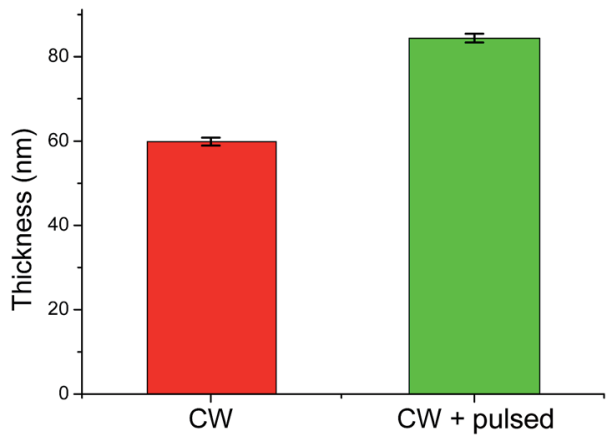

Fig. 1 Film thicknesses of AGEpp coatings as measured by ellipsometry.

ethanol and acetone was unsurprising, as previous studies have shown that these solvents can remove soluble materials from plasma polymers. ${ }^{13}$ A gentler alternative was needed, which could still remove possibly present non-covalently bound caspofungin (and other molecules of interest for bio-conjugation onto AGEpp), which might not be achieved by washing with water alone. For this reason, we investigated washing samples with aqueous sodium dodecyl sulphate (SDS) at $70{ }^{\circ} \mathrm{C}$ for at least half an hour. This method led to only a $5 \%$ decrease in thickness for the CW coating and $12 \%$ for the $\mathrm{CW}+$ pulsed coating. Hence, we chose, after caspofungin immobilisation, to wash samples with SDS at elevated temperatures. Previously we investigated the roles of physisorption and chemisorption for caspofungin adsorption to plasma polymer surfaces. Surface analysis on positive and negative control samples provided convincing evidence for covalent attachment of caspofungin after using these washing conditions and also did not affect the biological efficacy of the remaining covalently bound molecules. ${ }^{14}$ Furthermore, when producing coatings on an industrial scale it would be much preferable ecologically to use SDS washing rather than organic solvents.

Thus, AGEpp coatings were exposed to caspofungin in phosphate buffered saline (PBS) and then subjected to SDS washing. As shown in Fig. 3, nitrogen is absent on AGEpp coatings, but nitrogen (2.2\%) appears on the AGEpp

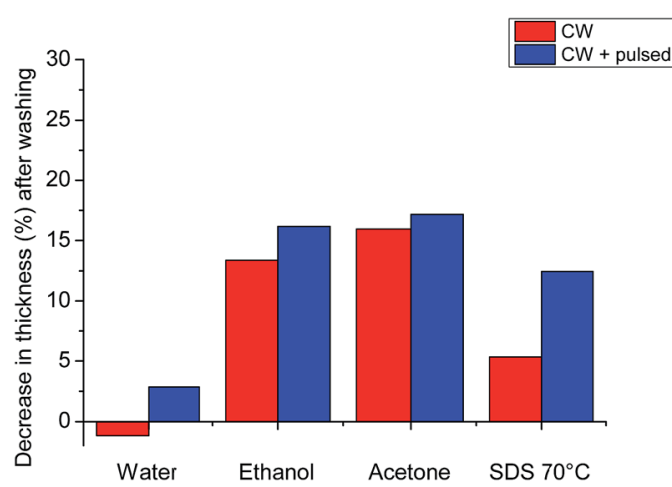

Fig. 2 Decrease in plasma polymer coating thickness due to exposure to different solvents and conditions. Positive values represent a percentage thickness decrease relative to the dry coating thickness. Error bars are present but appear small at this scale. 
caspofungin-grafted and SDS washed surfaces. As caspofungin contains nitrogen and AGEpp does not, this is an indication that caspofungin has been bound to the surface; analogous to a previous study using different interfacial conjugation chemistry but the same washing conditions. ${ }^{\mathbf{1 4}}$ Furthermore, judging from the nitrogen content, the present AGEpp bound similar amounts of caspofungin as had previously been immobilised onto aldehyde plasma polymer interlayers $(2.6 \% \mathrm{~N}) .^{4}$

To assess the antifungal activity of the grafted caspofungin, we tested the surfaces using a modified method based on Chandra et al. (see ESI $\dagger$ ). ${ }^{15}$ This assay enables comparison of different antifungal surfaces and produces quantitative results. Results, shown in Fig. 4, reveal a marked difference in activity, demonstrating that the surface-grafted caspofungin is active, and this is further supported by kinetic fluorescent microscopy (Fig. 5).

Fig. 4 illustrates the growth of C. albicans (ATCC 90028) and C. glabrata (ATCC 90030) (both supplied by Dr Sarah Kidd of the National Mycology Reference Centre, SA Pathology, Adelaide, South Australia) on the AGEpp, on caspofungin-grafted samples, and SDS washed samples. Growth was significantly lower on the caspofungin-grafted surfaces when compared to the AGEpp surfaces. In the case of $C$. albicans, the reduction was greater than $\log 6$, while with $C$. glabrata there was a reduction of $\log 3$. This result is consistent with a previous report on the activity of a tethered caspofungin coating against $C$. glabrata which utilised a contact based assay to determine activity, ${ }^{\mathbf{1 4}}$ rather than the biofilm quantitation method used here.

Of note is that the antifungal results presented in Fig. 4 were obtained on surfaces that had first been exposed to bovine serum albumin (BSA, $0.1 \mathrm{mg} \mathrm{ml}{ }^{-1}$ ) for $24 \mathrm{~h}$ at $37^{\circ} \mathrm{C}$ to allow for protein physisorption. This step was included to understand the potential role of protein fouling on bioactive surface coatings as some have noted a loss of surface activity due to BSA surface fouling. ${ }^{16}$ Results show that the caspofungin-grafted surfaces remained antifungal despite protein fouling. The reason as to why BSA surface fouling does not seem to impair the efficacy of caspofungin-grafted surface coatings is a matter currently under investigation. It may be related to the way fungal cells attach to and spread across surfaces, as well as their associated virulence factors. Possibly BSA molecules are displaced off the surface as fungal cells compete for surface adsorption, re-exposing caspofungin. This needs be examined in more detail.

\begin{tabular}{|c|c|c|c|}
\hline & $\begin{array}{l}\% \\
\mathrm{C}\end{array}$ & $\% 0$ & $\begin{array}{l}\% \\
\mathbf{N}\end{array}$ \\
\hline AGEpp & 74.4 & 25.6 & - \\
\hline $\begin{array}{c}\text { AGEpp } \\
+ \text { Caspo } \\
\text { + SDS } \\
\text { wash }\end{array}$ & 69.6 & 26.7 & 2.2 \\
\hline
\end{tabular}

Fig. 3 Atomic composition in \% as determined by XPS. Experimental uncertainties are $<10 \%{ }^{1}$

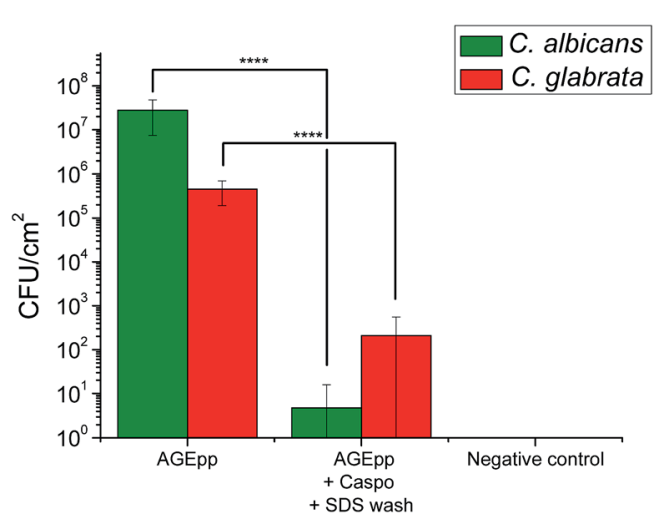

Fig. 4 Candida albicans and Candida glabrata growth on AGEpp and AGEpp + Caspo samples that had been incubated for $24 \mathrm{~h}$ in BSA (Sigma-Aldrich) solution.

As the interfacial conjugation reaction forms an amine bond (Scheme 1) and caspofungin contains multiple amine groups, it is not possible to acquire direct XPS spectral evidence for the formation of the interfacial bond. To verify that caspofungin was indeed covalently bound to the surface and not leaching into solution (at least to concentrations at the MIC or higher),

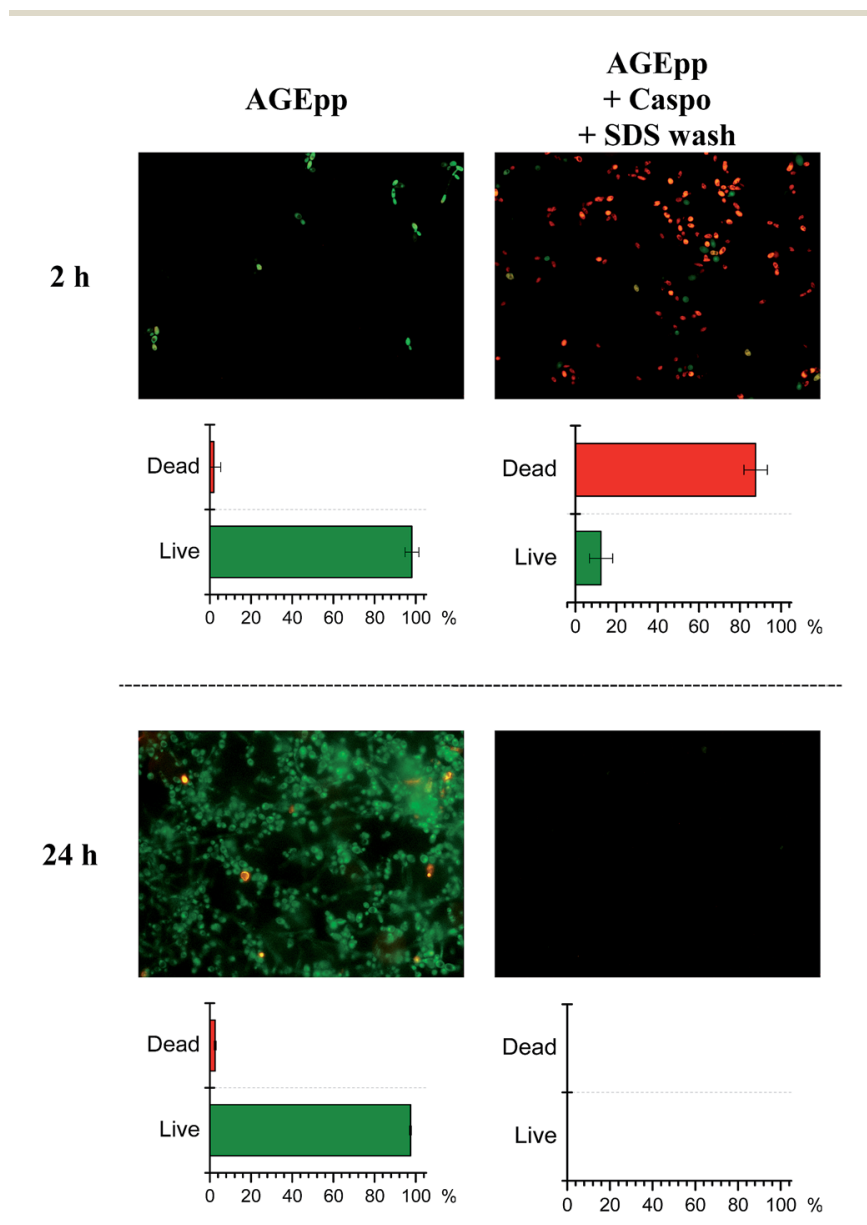

Fig. 5 Live-dead $(40 \times)$ stained micrographs and percentages of $C$. albicans in contact with the control and antifungal surfaces at 2 and $24 \mathrm{~h}$ time points. At $24 \mathrm{~h}$, the caspofungin modified surface yielded no visible cells to count. 
we counted the CFU of $C$. albicans in the supernatant after 90 minutes of incubation (Fig. S1†). Comparison of the AGEpp and the caspofungin modified surfaces showed that the numbers of planktonic cells in the supernatant were identical (within experimental uncertainty). This indicated that there was no measurable effect assignable to leaching of caspofungin killing fungal cells in solution above the samples.

To visualise the attachment and metabolic activity of cells on the surfaces, live-dead staining at set time intervals was conducted. The micrographs in Fig. 5 show C. albicans growth on AGEpp at both time-points, whereas a significant reduction of attachment, as well as a considerable extent of cell death, are seen on the caspofungin surfaces. After $2 \mathrm{~h}, 88 \%$ of fungal cells that were in contact with the caspofungin grafted surface were dead, while $98 \%$ of cells on the AGEpp were alive. By $24 \mathrm{~h}$ no detectable numbers of $C$. albicans were present on the caspofungin-grafted surface, whereas on the AGEpp surface fungal cells had developed a mature biofilm containing cells with hyphal phenotype. Observations in-between these timepoints (after 4, 6 and 8 hours), are depicted in Fig. S2 $\uparrow$ and illustrate the progressive formation of biofilm on the AGEpp surface and the contrasting death and eventual detachment of cells in contact with the caspofungin-grafted surface.

In summary, this study has demonstrated a convenient and straight-forward method for covalently binding the antifungal drug caspofungin from aqueous solution onto solid materials surfaces. The antifungal drug and the polymer coating were covalently attached and had excellent solvent stability. Caspofungin-grafted surfaces were very efficient against the clinically significant fungal pathogens $C$. albicans and C. glabrata, even when samples were pre-conditioned with bovine serum albumin. By using an epoxide plasma polymer coating that enables one-step covalent attachment and does not require a reducing agent, facile production of covalently grafted antifungal surfaces can be achieved, suitable for the industrial manufacture of antifungal surface coatings for medical devices. Similar coating processes for other health-care devices have resulted in commercially successful functional biomaterials, for example in the case of the plasma coating used on Ciba Vision Night \& Day contact lenses. ${ }^{17}$ The mechanism of resistance to biofouling, activity against a broader variety of pathogenic Candida species, biocompatibility and in vivo results will be reported in a follow-up study.

\section{Acknowledgements}

This research was supported through the Australian Research Council's Discovery Projects funding scheme (project DP DP150101674).

\section{Notes and references}

1 P. J. Cumpson and M. P. Seah, Surf. Interface Anal., 1992, 18, 361.
2 G. D. Brown, D. W. Denning and S. M. Levitz, Science, 2012, 336, 647.

3 (a) S. G. Whaley, E. L. Berkow, J. M. Rybak, A. T. Nishimoto, K. S. Barker and P. D. Rogers, Front. Microbiol., 2017, 7, DOI: 10.3389/fmicb.2016.02173; (b) A. Srinivasan, J. L. LopezRibot and A. K. Ramasubramanian, Drug Discovery Today: Technol., 2014, 11, 65; (c) D. M. Kuhn and M. A. Ghannoum, Curr. Opin. Invest. Drugs, 2004, 5, 186.

4 S. S. Griesser, M. Jasieniak, B. R. Coad and H. J. Griesser, Biointerphases, 2015, 10, $04 a 307$.

5 B. R. Coad, H. J. Griesser, A. Y. Peleg and A. Traven, PLoS Pathog., 2016, 12, e1005598.

6 D. W. Denning, Lancet, 2003, 362, 1142.

7 (a) S. Kucharíková, E. Gerits, K. De Brucker, A. Braem, K. Ceh, G. Majdič, T. Španič, E. Pogorevc, N. Verstraeten, H. Tournu, N. Delattin, F. Impellizzeri, M. Erdtmann, A. Krona, M. Lövenklev, M. Knezevic, M. Fröhlich, J. Vleugels, M. Fauvart, W. J. de Silva, K. Vandamme, J. Garcia-Forgas, B. P. A. Cammue, J. Michiels, P. Van Dijck and K. Thevissen, J. Antimicrob. Chemother., 2016, 71, 936; (b) A. Braem, K. De Brucker, N. Delattin, M. S. Killian, M. B. J. Roeffaers, T. Yoshioka, S. Hayakawa, P. Schmuki, B. P. A. Cammue, S. Virtanen, K. Thevissen and B. Neirinck, ACS Appl. Mater. Interfaces, 2017, 9, 8533.

8 G. T. Hermanson, in Bioconjugate Techniques, Academic Press, Boston, 3rd edn, 2013, pp. 259-273.

9 B. Thierry, M. Jasieniak, L. de Smet, K. Vasilev and H. J. Griesser, Langmuir, 2008, 24, 10187.

10 B. R. Coad, M. Jasieniak, S. S. Griesser and H. J. Griesser, Surf. Coat. Technol., 2013, 233, 169.

11 C. Tarducci, E. J. Kinmond, J. P. S. Badyal, S. A. Brewer and C. Willis, Chem. Mater., 2000, 12, 1884.

12 (a) J. Friedrich, Plasma Processes Polym., 2011, 8, 783; (b) M. E. Ryan, A. M. Hynes and J. P. S. Badyal, Chem. Mater., 1996, 8, 37; (c) D. Debarnot, T. Mérian and F. PoncinEpaillard, Plasma Chem. Plasma Process., 2011, 31, 217.

13 M. T. van Os, B. Menges, R. Foerch, G. J. Vancso and W. Knoll, Chem. Mater., 1999, 11, 3252.

14 B. R. Coad, S. J. Lamont-Friedrich, L. Gwynne, M. Jasieniak, S. S. Griesser, A. Traven, A. Y. Peleg and H. J. Griesser, J. Mater. Chem. B, 2015, 3, 8469.

15 J. Chandra, P. K. Mukherjee and M. A. Ghannoum, Nat. Protoc., 2008, 3, 1909.

16 (a) I. Banerjee, R. C. Pangule and R. S. Kane, Adv. Mater., 2011, 23, 690; (b) K. Nakanishi, T. Sakiyama and K. Imamura, J. Biosci. Bioeng., 2001, 91, 233.

17 P. C. Nicolson, R. C. Baron, P. Chabrecek, J. C. A. Domschke, H. J. Griesser, A. Ho, J. Hopken, B. G. Laycock, Q. Liu, D. Lohmann, G. F. Meijs, E. Papspiliotopoulos, J. S. Riffle, K. Schindhelm, D. Sweeney, W. L. Terry, J. Vogt and L. C. Winterton, US Pat., US2005228065, 2005. 